\title{
Experimental Demonstrations of Real-time MILP Control
}

\author{
Arthur Richards*, Yoshiaki Kuwata, ${ }^{\dagger}$ and Jonathan How $^{\ddagger}$ \\ MIT Dept. of Aeronautics and Astronautics ${ }^{\S}$
}

\begin{abstract}
This paper presents results from recent hardware experiments using two forms of on-line Mixed-integer Linear Programming (MILP). The demonstrations were performed on wheeled ground vehicles, with a view to future use on autonomous teams of UAVs. The first experiment uses MILP at a high-level to account for a limited detection horizon while maneuvering in the presence of obstacles. The second performs low-level control with MILP to reject disturbances while performing a rendezvous. The paper describes the design of the multi-vehicle testbed and discusses the architecture and implementation details of the experiments. In both cases, the online MILP compensated for the uncertainties present and successfully completed the maneuvers.
\end{abstract}

\section{INTRODUCTION}

Two forms of predictive control based on Mixedinteger Linear Programming (MILP) are demonstrated on a multi-vehicle testbed. The objective of these controllers is to enable coordinated operation of multiple Unmanned Aerial Vehicles (UAVs) to achieve high-level mission goals in a challenging environment [1]. The experiments, performed using wheeled ground vehicles to emulate the UAVs, highlight the transition from simulation to hardware implementation. A key issue is the interaction between highand low-level controllers within the overall control system architecture. Significant dynamic uncertainty is present, ranging from disturbance forces acting on the vehicles to macroscopic changes in the environment, such as the discovery of new targets or obstacles. The two demonstrations in this paper show how MILP can be applied at different levels of the control system to account for these different types of uncertainty.

\footnotetext{
* Research Assistant, arthurr@mit.edu

$\dagger$ Research Assistant, kuwata@mit.edu

¥ Associate Professor, jhow@mit.edu, Senior Mbr. AIAA.

$\S$ MIT 33-328, 77 Mass. Ave., Cambridge, MA 02139.
}

Previous work has demonstrated the use of MILP for off-line trajectory design for vehicles subject to avoidance constraints $[4,7,8]$. MILP enables the inclusion of non-convex constraints and discrete decisions in the trajectory optimization. Binary decision variables allow the choice of whether to pass "left" or "right" of an obstacle, for example, or the discrete assignment of vehicles to targets, to be included in the planning problem. MILP can also be used on-line, replanning in real-time to account for dynamic uncertainty. This is known as either Model Predictive Control (MPC) or receding horizon control [10]. In particular, the use of on-line MILP for vehicle path-planning with avoidance has been proposed in two forms. In one, a MILP optimization is performed over a shortened horizon, with the cost-to-go represented by a realistic path approximation [2]. In this paper, this will be referred to as the cost-to-go method. In the other form, known here as the whole trajectory method, the entire trajectory is redesigned [10]. More recent developments of the latter scheme include a variable terminal time, which offers stability for a general target state, and modifications for robustness [5]. Both methods have been considered analytically and in simulation. The innovation of this paper is their implementation with hardware in the loop, including the details of application and the architectures used.

MPC has been successfully applied to chemical process control [6]. However, application to systems with faster dynamics, such as those found in the field of aerospace, has been impractical until recently, with the on-going improvements in computation speed. Recent hardware work in this field includes the online use of NTG nonlinear optimization for control of an aerodynamic system $[11,12]$ and of a formation of highly-nonlinear vehicles $[13,14]$.

The testbed used in this work consists of remotecontrolled, wheel-steered miniature trucks. For these experiments, they are operated at constant speed. Due to limited steering angles, their turn rate is restricted. Therefore, the trucks can be used as repre- 
sentative models of UAVs, which would typically operate at a constant, nominal speed, flying at a fixed altitude and with turning rate limited by the achievable bank angle. The vehicles have on-board computers and wireless LAN communications, offering a flexible architecture for demonstrating co-operative control.

In the first demonstration, the cost-to-go method [2] is used as a "high-level" controller to compensate for uncertainty in the environment. It designs a series of waypoints for the truck to follow. A "low-level" controller then steers the truck to move along this path. An obstacle exists between the vehicle and its goal, but the controller does not become aware of this until it comes within a "detection horizon" of the obstacle. This represents operation using sensors with limited range, such as radar, which can detect obstacles only within a certain radius.

In the second demonstration, the low-level controller is removed and the on-line MILP is used to compensate for disturbances acting upon the vehicle. The whole-trajectory formulation is used [5], including modifications to ensure feasibility of the MILP at each step. Two vehicles are used: one is driven open-loop as a target while the other is controlled by MPC to perform a rendezvous with the first. The MPC must compensate for uncertain motion of both trucks. For the purposes of the experiments, the choice of a rendezvous maneuver adds the unknown target motion to the sources of uncertainty. In practice, such a maneuver could represent in-flight refuelling of a UAV.

This paper begins with a description of the truck testbed used, which is common to both demonstrations. Each is then discussed separately, including specific objectives, controller formulations, integration with the testbed, test scenarios, procedures and results.

\section{APPARATUS DESCRIPTION}

The testbed for this experiment consists of two selfcontained, remotely-controlled trucks. They can be operated in two distinct modes. Fig. 1(a) shows the architecture and loop closure in waypoint mode, in which the high-level controller provides a set of waypoints to the trucks and a low-level controller moves between them. In direct steer mode, shown in Fig. 1(b), the high-level controller generates steering commands for the truck, while a low-level controller maintains a desired speed. The major components are described separately in the following sections.

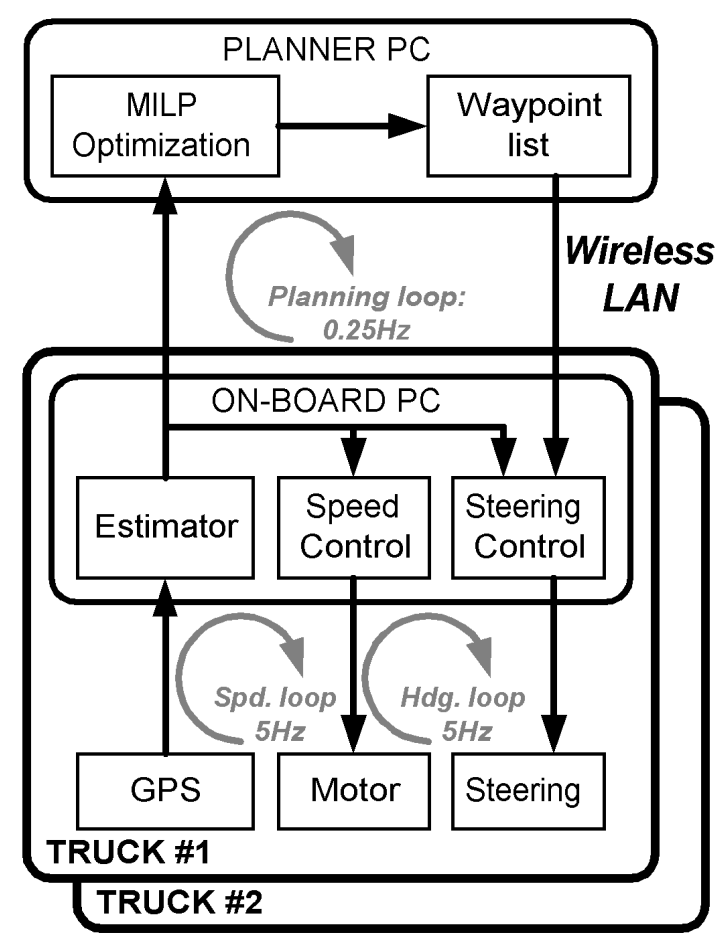

(a) Waypoint Mode

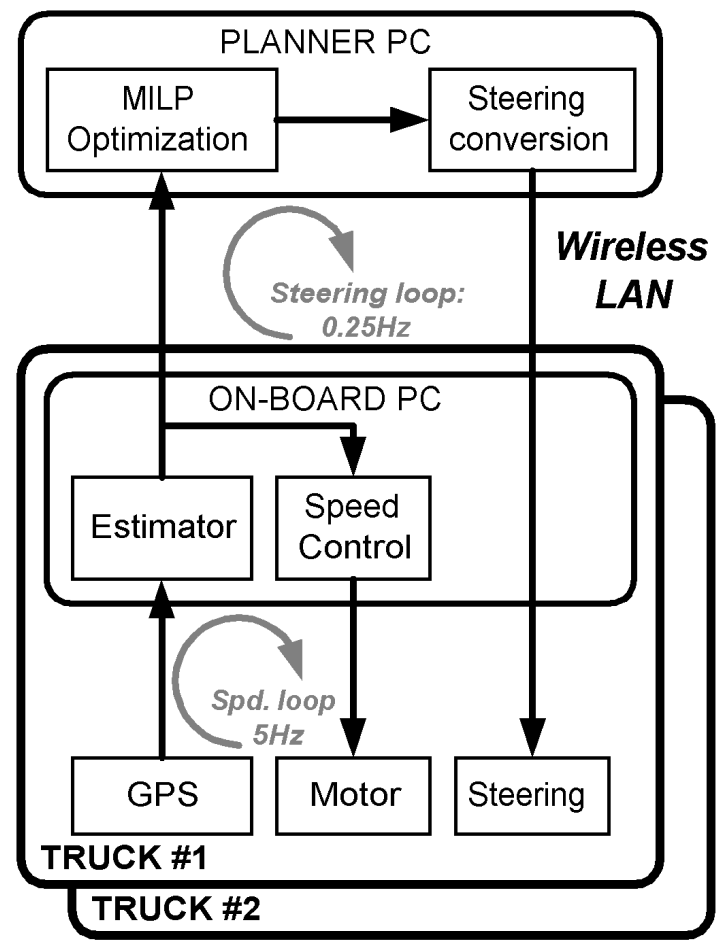

(b) Direct Steer Mode

Fig. 1: Testbed Architecture showing Control Loops in Both Modes 


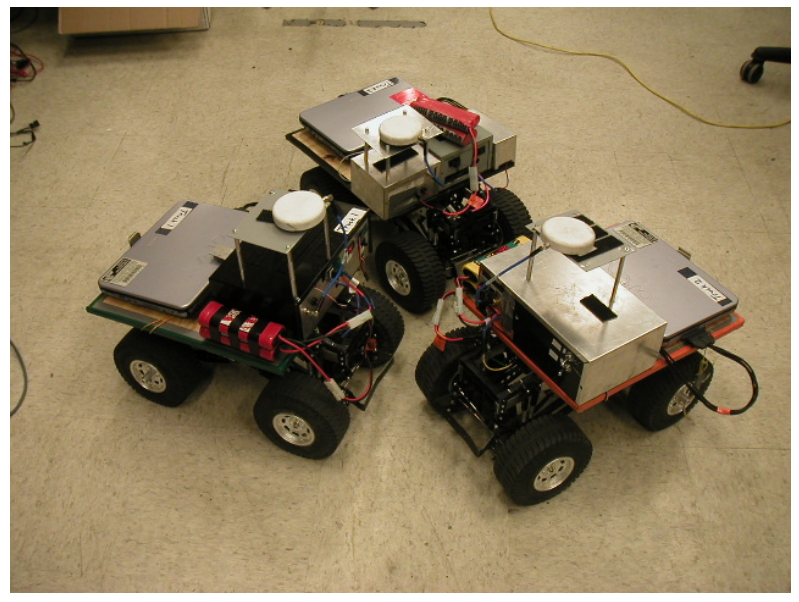

Fig. 2: Testbed vehicles.

Trucks

Fig. 2 shows three of the trucks. Each is based on a commercially available model truck. Each carries a laptop PC on-board, the functions of which are described in the next section. Physically, it is connected by serial links to a PWM converter, generating signals for the motor speed controller and steering servo, and to a GPS receiver. The batteries and power supply circuitry complete the on-board hardware, making each truck an independent unit. A video camera can also be mounted on-board for demonstration purposes.

\section{On-Board Computer}

This laptop computer on each truck performs local estimation and low-level control functions. It performs precise relative position and velocity estimation using differential carrier-phase GPS [17]. A fixed GPS base station, not shown in Fig. 1, communicates with the on-board PC via wireless LAN to enable differential GPS. The estimation is performed at a rate of $5 \mathrm{~Hz}$. A speed control loop is closed on the laptop, generating motor servo commands in order to maintain a constant reference speed. In waypoint mode, an additional steering controller acts to follow a list of waypoints. In direct steer mode, the steering servo command is relayed directly from a higher-level controller. The higher level commands (reference speed, waypoint list and steering command) are sent via wireless LAN from the planner computer. To close the loop, the GPS state estimates are sent to the planner at a rate of $1 \mathrm{~Hz}$.

\section{Planner}

This laptop computer performs the MILP optimizations. In waypoint mode, the first few waypoints of the plan are sent to the base-station. In directsteer mode, a steering angle is computed for the first plan step and sent to the base-station for direct relay to the truck. MATLAB is used to manage communications, data recording, visualization and data conversion. The planner uses CPLEX optimization software [16] to solve the MILP problems, using AMPL [15] as the interface between CPLEX and MATLAB.

\section{Operating Limits}

To achieve as much as possible in a confined space, the trucks are operated at a low speed (nominal running speed is $0.5 \mathrm{~m} / \mathrm{s}$ ). At lower speeds, friction effects made the speed control unreliable. At this speed, the available steering range sets the minimum turn radius to approximately $1 \mathrm{~m}$.

\section{COST-TO-GO DEMONSTRATION}

In these experiments, the receding-horizon MILP formulation with the approximate cost-to-go function [2] is used to compensate for uncertainty in the obstacle environment beyond a limited detection horizon. In this case, disturbances acting on the truck are assumed to be handled by the lowlevel feedback control system. The detection radius effect is simulated, since the actual trucks have no real obstacle sensors. For simplicity, the controller is given the size and location of the complete obstacle as soon as its detection radius intersects part of the obstacle [3].

The following subsection reviews the receding horizon formulation. In the final subsection, the results are presented and interpreted.

\section{Review of Receding Horizon Control}

For review, the cost-to-go formulation for the minimum time path planning problem is briefly presented here [2]. This algorithm designs the minimumtime path to a fixed goal while avoiding a known set of obstacles. Operation in the presence of the limited detection horizon is described in the next subsection. Fig. 3 gives an overview of the method, including the different levels of resolution involved.

The control strategy is comprised of two phases: cost estimation and trajectory design. The cost estimation estimates the cost-to-go from each obstacle corner. The procedure is as follows:

1. Form a graph by connecting every obstacle corner to all other obstacle corners using straight lines. 


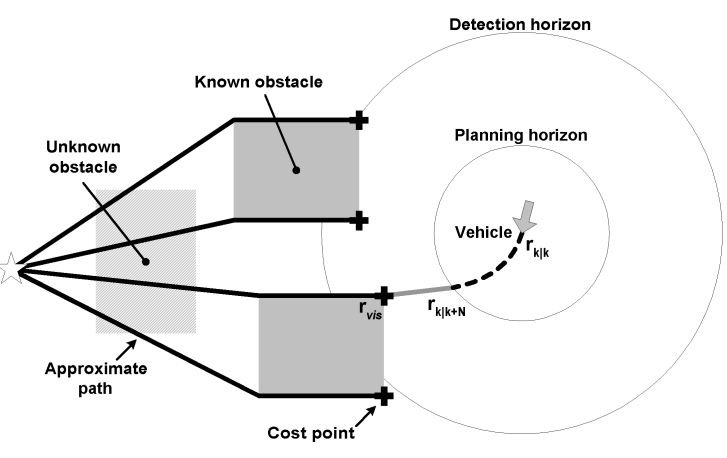

Fig. 3: Overview of Cost-to-Go Method.

2. Remove those edges that intercept obstacles.

3. Use Dijkstra's Single-Source Shortest-Path Algorithm to find the "tree" of optimal paths from the goal to each corner.

4. Store the cost-to-go from each corner along the calculated path.

The development of this set of approximate costs is based on the observation that the true optimal paths (i.e. minimum distance) tend to follow the edges and corners of the obstacles. In Fig. 3, each of the "cost points" is marked by + and the associated paths are the solid black lines. Note that it is not necessary to store the paths: only the cost values are needed.

In the trajectory design phase, MILP optimizations are solved to design a series of short trajectory segments over a planning horizon with length $N$ steps. In Fig. 3, this section of the plan is shown by the dashed black line. Each optimization finds a control sequence $\left\{\mathbf{u}_{k \mid k} \ldots \mathbf{u}_{k \mid(k+N-1)}\right\}$ and corresponding states $\left\{\mathbf{x}_{k \mid k} \ldots \mathbf{x}_{k \mid(k+N)}\right\}$, where $\mathbf{u}_{k \mid j}$ denotes the control designed at time $k$ for application at time $j$ and each state is comprised of a position and velocity i.e. $\mathbf{x}=(\mathbf{r} \mathbf{v})$. These states are subject to the dynamics constraint

$$
\forall j \in[k \ldots(k+N-1)] \mathbf{x}_{\mathrm{k} \mid(\mathrm{j}+1)}=\mathbf{A} \mathbf{x}_{\mathrm{k} \mid \mathrm{j}}+\mathbf{B} \mathbf{u}_{\mathrm{k} \mid \mathrm{j}}
$$

where $\mathbf{A}$ and $\mathbf{B}$ are the discretized system dynamics matrices for a point mass in 2-D free-space. The speed and force are limited, using the following approximation for a 2-norm [8]

$$
\begin{aligned}
& \forall j \in[k \ldots(k+N)] \mathbf{W} \mathbf{v}_{k \mid j} \leq \mathbf{1} v_{\max } \\
& \forall j \in[k \ldots(k+N)] \mathbf{W} \mathbf{u}_{k \mid j} \leq \mathbf{1} f_{\max }
\end{aligned}
$$

where $\mathbf{1}$ is a vector of 1's and $\mathbf{W}$ has the structure

$$
\mathbf{W}=\left[\begin{array}{cc}
\cdots & \cdots \\
\cos \left(\theta_{\mathrm{i}}\right) & \sin \left(\theta_{\mathrm{i}}\right) \\
\cdots & \cdots
\end{array}\right]
$$

The values of $\theta_{\mathrm{i}}$ are uniformly distributed between 0 and $2 \pi$. By limiting the components of the vector quantities in each of these directions, an approximate limit on their two-norms is effected in linear form [8]. A point mass dynamic model subject to the 2-norm force and speed constraints (2) and (3) forms a good approximate model for limited turnrate vehicles, provided that the optimization favors the minimum time, or minimum distance, path [8].

The MILP also chooses a "visible point" $\mathbf{r}_{\text {vis }}$ which is visible from the "terminal point" $\mathbf{r}_{k \mid k+N}$ and from which the cost-to-go has been estimated, $C_{\text {vis }}$, in the previous phase. These points are shown in Fig. 3. Note that the cost map is quite sparse: cost-to-go values are only known for the corners of the obstacles. The distance from the terminal point to the visible point is approximated in the cost function, allowing the cost-to-go to be evaluated at a freelychosen terminal point using only a sparse grid of cost points. Since the optimal trajectories usually pass through obstacle vertices, this is a valid approximation and the performance remains close to the true optimal [2]. The map does not need to be recalculated at each step: it is valid as long as the obstacles are known and stationary. Changes in the obstacle field are dealt with by replanning, as discussed in the next section.

The following avoidance constraints are applied at each point of the dynamic segment and at intermediate points between the terminal point and the visible point. Rectangular obstacles are used in this formulation, and are described by their lower left corner $\left(u_{\text {low }}, v_{\text {low }}\right)$ and upper right corner $\left(u_{\text {high }}, v_{\text {high }}\right)$. To avoid collisions, the following constraints must be satisfied at each point on the trajectory [9]

$$
\begin{aligned}
r_{k \mid j, 1} & \leq u_{\text {low }}+R b_{\text {in }, 1} \\
r_{k \mid j, 1} & \geq u_{\text {high }}-R b_{\text {in }, 2} \\
r_{k \mid j, 2} & \leq v_{\text {low }}+R b_{\text {in }, 3} \\
r_{k \mid j, 2} & \geq v_{\text {high }}-R b_{\text {in }, 4} \\
\sum_{\mathrm{j}=1}^{4} b_{\text {in }, \mathrm{j}} & \leq 3
\end{aligned}
$$

The trajectory cost involves three terms: the cost of the steps to the terminal point $\mathbf{r}_{k \mid k+N}$; the approximate straight-line cost from the terminal point to the visible point $\ell_{\text {vis }}=\left\|\mathbf{r}_{k \mid k+N}-\mathbf{r}_{\text {vis }}\right\|$; and the cost from the visible point to the goal $C_{\text {vis }}$. Referring to Fig. 3, these represent the black dashed line, the gray line, and the black solid line, respectively. Assuming that the first part of the plan is flown at 
constant speed, its distance is constant and it can be ignored in the cost function. This assumption is valid for the minimum distance, limited speed problem [8]. Only the distance to the visible point and the remaining straight-line approxmate path are included. The actual cost function is

$$
J^{*}=\min _{\mathbf{u}(\cdot)}\left[\frac{\ell_{\mathrm{vis}}}{v_{\max }}+C_{\mathrm{vis}}\right]
$$

with Eq. (1) - (6) as constraints. The following constraints determine $\ell_{\text {vis }}$

$$
\mathbf{W}\left(\mathbf{r}_{k \mid k+N}-\mathbf{r}_{\mathrm{vis}}\right) \leq \mathbf{1} \ell_{\mathrm{vis}}
$$

This approximates the two-norm of the distance from the terminal point to the visible point, by approximating the smallest circle to enclose that vector.

\section{Detection Horizon Application}

This section describes the application of the formulation in the previous section to the truck demonstration. The truck was modeled as having maximum speed $0.5 \mathrm{~m} / \mathrm{s}$, equal to the nominal running speed. The modeled maximum turn rate was $7 \mathrm{deg} / \mathrm{s}$, chosen to give smooth operation when using the lowlevel steering controller: the planner sends waypoints rather than steering commands in this demonstration. The system was discretized with a time-step of four seconds, long enough for the transient of the low-level steering controller to decay. The planning horizon was eight steps, equivalent to up to $25.6 \mathrm{~m}$. The execution horizon was one step: after each plan was found, only one new waypoint was uploaded to the truck.

The initial condition for each new plan is taken as the first waypoint of the previous plan. This allows the next plan to be made before the execution point is reached, reducing the control delay to that required for communication. This demonstrates the two-level architecture of the detection horizon demonstration. It is the responsibility of the lowlevel steering controller to see that the vehicle reaches the prescribed waypoint. The high-level MILP controller closes a different real-time loop, as it compensates for obstacle information that only becomes available as the vehicle moves.

The detection horizon was $16 \mathrm{~m}$. Initially, the cost map is computed using only those obstacles within the detection horizon of the starting point. At each step, before the trajectory design phase, the circle enclosed by the detection horizon around the current position is checked for intersections with new obstacles. If any are "found," the cost estimation is repeated including the new obstacle. Note that this assumes that complete knowledge of the obstacle is available as soon as part of it is detected. On-going research is investigating how to include partiallydetected obstacles in the path planning [3].

In both the cost estimation and trajectory design phases, it is necessary to include an enlarged model of the obstacle. Since the avoidance constraints are only applied at discrete time steps, it would be possible for the planned trajectory to "cut the corner" of the obstacle between time points. To prevent collisions with the real obstacle, the obstacle models are $\left(v_{\max } \Delta t / \sqrt{2}\right)$ larger in each direction. This is the maximum incursion distance.

In summary, the control scheme for the cost-to-go method is as follows:

1. If the detection circle intersects any "new" obstacles (or if starting), compute the cost map, including all obstacles currently known.

2. Solve MILP (7) subject to (1)-(6) and (8), starting from the last waypoint downloaded (or initial state if starting).

3. Upload the first waypoint $\mathbf{r}_{\mathrm{k} \mid \mathrm{k}+1}$ of the new plan to the truck

4. Wait until truck reaches the execution horizon from the current point.

5. Go to 1 .

\section{Detection Horizon Tests}

The test scenario is shown in Fig. 4(a). The truck begins at the origin $(0,0)$, heading in the direction of the goal $(-50,-20)$. A single obstacle, marked by the solid black line, blocks the direct path to the goal. However, it is beyond the detection horizon (shown by the large circle) and hence not initially known to the planner. The obstacle was marked by cones in the test area. To successfully complete the test, the vehicle must move around the obstacle, once it is aware of its presence, and reach the goal, heading in the $-\mathrm{x}$ direction.

When the experiment was performed, the truck was observed to begin by heading straight to the goal. When it approached the obstacle, it turned from the straight path and went around the cones marking the obstacle. It then proceeded straight to the goal. Plots of the result are shown in Fig. 4. In Fig. 4(a), the first plan from initial position over the execution horizon has been uploaded to the truck controller. Since it is not aware of the obstacle, the planner has commanded a path directly towards the goal. The 


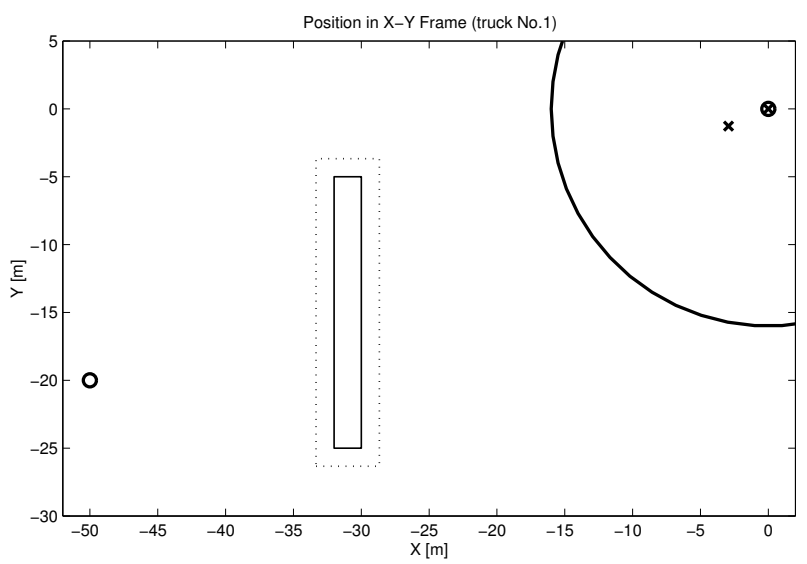

(a)

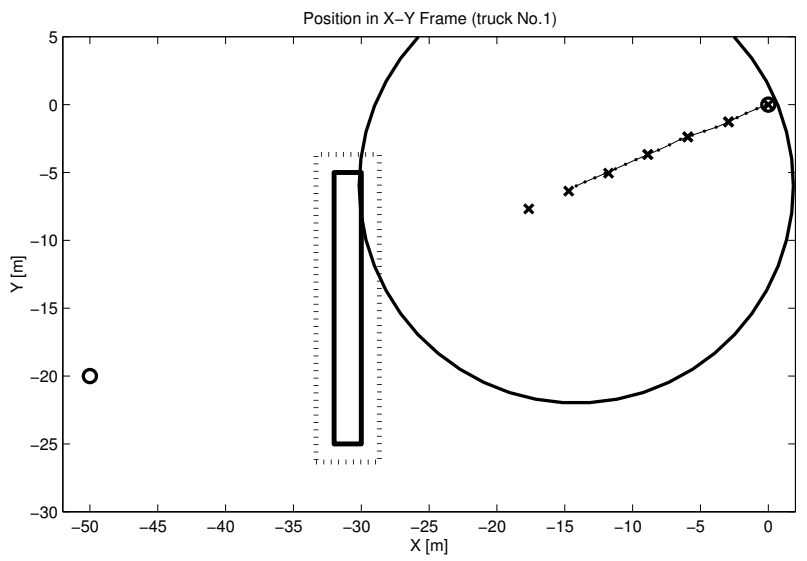

(c)

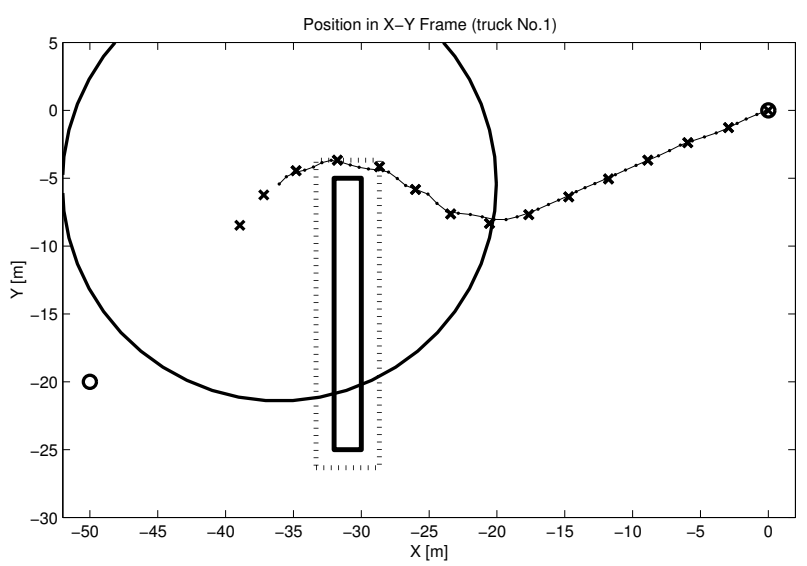

(e)

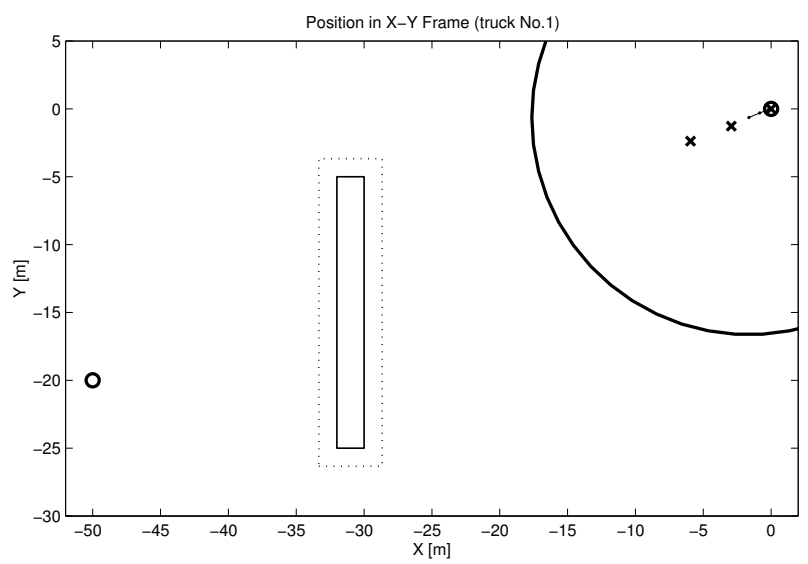

(b)

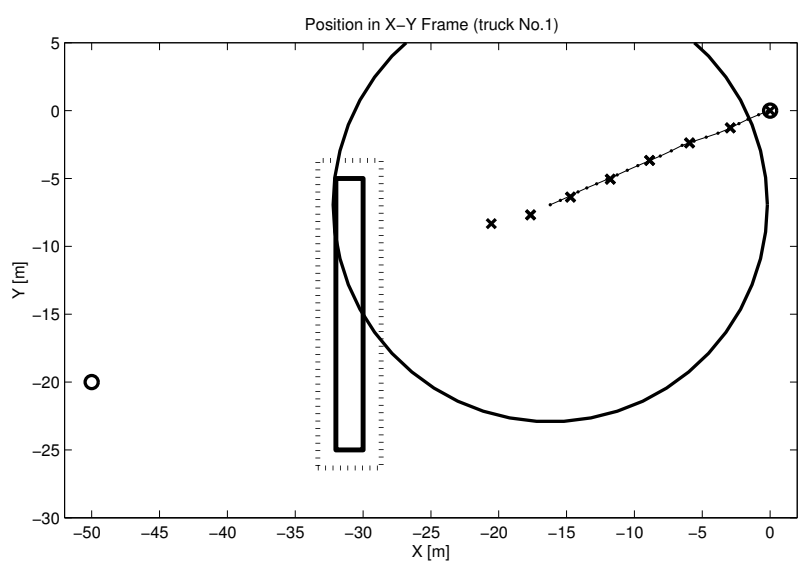

(d)

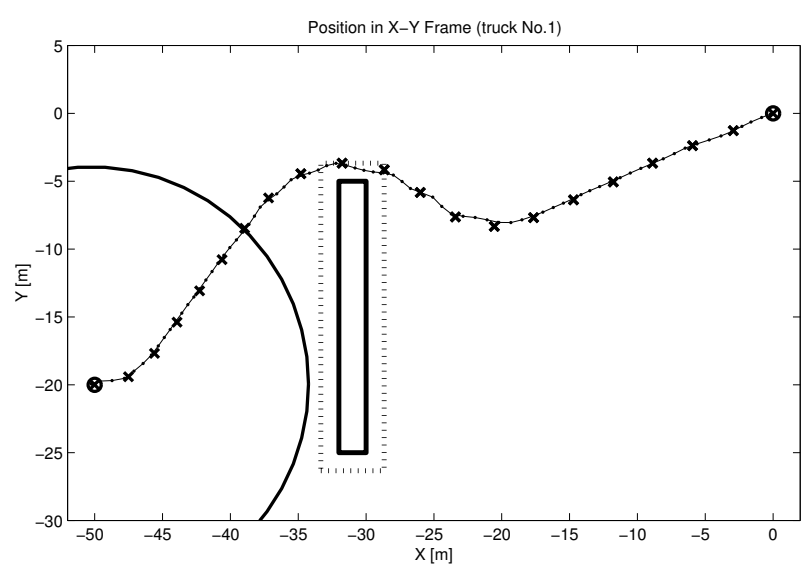

(f)

Fig. 4: The trajectory generated by planner and the experimental data of the actual trajectory of the truck. The circle shows the detection horizon. 
truck starts moving and the planner starts solving for the next plan, starting from the execution horizon of the previous plan.

Two seconds later, shown in Fig. 4(b), the truck is about to reach the waypoint sent from the first plan. Having completed the new plan, the next waypoint has been uploaded. The obstacle has not yet been detected, and the designed path is still straight to the goal.

In Fig. 4(c), the obstacle is detected when it comes within the detection horizon of the truck. The next waypoint is uploaded, still heading straight to the goal, since the last plan was not aware of the obstacle. However, the planner now recomputes the cost map, including the obstacle, before making the next plan. The new map includes the enlarged obstacle, shown by the dashed line. In Fig. 4(d), the next plan reflects the new obstacle information and leads the truck to go around (above in the figure) the obstacle.

Figs. 4(e) and 4(f) show the resulting trajectory to the goal. At the end, the truck aligns its heading with $-x$ direction as is specified in the terminal condition.

These initial results show that the cost-to-go method can control a real vehicle in an uncertain environment. The control architecture allows the online planner to compensate for obstacle discovery beyond the execution horizon while low-level feedback rejects vehicle disturbance within that horizon.

\section{MPC FOR RENDEZVOUS}

In these experiments, the low-level feedback controller is removed. One truck is driven open-loop ("the target") while the other ("the chaser") is controlled by the whole trajectory MPC to rendezvous with the target. The MPC must now compensate for uncertainties including model error, disturbances and the uncertain motion of the target truck. The formulation used for these experiments was introduced in Ref. [5]. It is reviewed briefly here, followed by specific details related to these experiments, before the results are presented.

\section{Controller Formulation}

The controller in this section uses the whole-trajectory MPC formulation described in Ref. [5]. It has been shown to guarantee maneuver completion in finite time. It is presented in brief form here. As in the cost-to-go method, the core of the problem is to design a sequence of states $\mathbf{x}$ and controls $\mathbf{u}$ subject to the point mass dynamics constraints (1)-(3). However, in contrast to the previous method, there is no cost-to-go at the end of the horizon, and the maneuver must be completed before the $N^{\text {th }}$ step. "Completion" is achieved when the states are steered into the region defined by $\mathbf{P x}_{\mathbf{x}} \leq \mathbf{q}$. Typically $\mathbf{P}$ and $\mathbf{q}$ define a position box, requiring the vehicle to be within some distance of a target point. They may also apply velocity constraints as well, such as some maximum velocity at the finish or some prescribed direction of travel.

There are additional discrete (binary) decision variables in this formulation: an 'input'

$$
\left\{v_{k \mid k} \ldots v_{k \mid(k+N-1)}\right\}
$$

and a 'state'

$$
\left\{y_{k \mid k} \ldots y_{k \mid(k+N)}\right\}
$$

for which $y_{\mathrm{k}}=0$ is defined to mean that the maneuver has been completed at or before step $k$. The following two constraints define a state machine for the discrete state $y$ and coupling with the continuous states:

$$
\begin{gathered}
\forall j \in[k \ldots(k+N-1)] \\
y_{\mathrm{k} \mid(\mathrm{j}+1)}=y_{\mathrm{k} \mid \mathrm{j}}-v_{\mathrm{k} \mid \mathrm{j}} \\
\mathbf{P}\left(\mathbf{A x}_{\mathrm{k} \mid \mathrm{j}}+\mathbf{B u}_{\mathrm{k} \mid \mathrm{j}}\right) \leq \mathbf{q}+\mathbf{1} M\left(1-v_{\mathrm{k} \mid \mathrm{j}}\right)
\end{gathered}
$$

where $M$ is a large positive number. If $v_{\mathrm{k} \mid \mathrm{j}}=1$, then (9) implies $y$ transitions from 1 at step $j$ to 0 at step $j+1$ while (10) implies that the states are in the target region at step $j+1$.

The cost function is a combination of the maneuver time and a low weighting on the applied force

$$
J^{*}=\min _{\{\mathbf{x}, \mathbf{u}, y, v\}} \sum_{\mathrm{j}=\mathrm{k}}^{(k+N-1)}\left(\epsilon\left|u_{\mathrm{k} \mid \mathrm{j}}\right|+y_{\mathrm{k} \mid \mathrm{j}}\right)
$$

where the small weighting $\epsilon \ll 1$ on force has been shown to help the solution process [18]. Since $y=1$ implies that the maneuver is not finished, the $y_{\mathrm{k} \mid \mathrm{j}}$ term included in the summation in (11) is effectively a penalty on maneuver time, in units of time steps.

The following constraints bound the approximated two-norm of the force input, with robustness modifications to tighten the limit in the far future

$$
\begin{aligned}
\mathbf{W u}_{k \mid k} & \leq \mathbf{1} f_{\max } \\
\mathbf{W u}_{k \mid k+1} & \leq \mathbf{1} f_{\max }-\beta_{1} \\
\mathbf{W} \mathbf{u}_{k \mid j} & \leq \mathbf{1} f_{\max }-\beta_{1}-\beta_{2} \forall j>k+1
\end{aligned}
$$


where the matrix $\mathbf{W}$ is defined in (4). Similar constraints are applied to the velocity

$$
\begin{aligned}
\mathbf{W} \mathbf{v}_{k \mid k} & \leq \mathbf{1} M \\
\mathbf{W} \mathbf{v}_{k \mid k+1} & \leq \mathbf{1} v_{\max }+\alpha \\
\mathbf{W} \mathbf{v}_{k \mid j} & \leq \mathbf{1} v_{\text {max }} \forall j>k+1
\end{aligned}
$$

Note that the constraint is completely relaxed on the first step, since this is fixed by the initial conditions. It was shown in Ref. [5] that, given a bound on the disturbances, values of $\alpha, \beta_{1}$ and $\beta_{2}$ can be chosen such that the MILP is guaranteed to be feasible under the action of the disturbance. If a solution exists at time step $k$, then it can be shown that a candidate solution, involving a two-step correction for the disturbance applied at $k$, is feasible at step $(k+1)$. Therefore, feasibility at the first step implies feasibility at all future steps.

Equations (12) and (13) are "operating constraints," applied at every step, and can be expressed in the general form

$$
\mathbf{C}_{1}(\mathrm{j}) \mathbf{x}_{\mathrm{k} \mid \mathrm{j}}+\mathbf{C}_{2}(\mathrm{j}) \mathbf{u}_{\mathrm{k} \mid \mathrm{j}}+\mathbf{C}_{3}(\mathrm{j}) \leq \mathbf{0}
$$

where $\mathbf{C}_{\mathrm{i}}(j)$ are derived from (12) and (13). For simplicity, avoidance constraints were not included in the rendezvous experiments. They can be applied if necessary by the inclusion of auxiliary binary vectors in the operating constraints. To guarantee finitetime completion, the operating constraints must be relaxed in the plan after the projected time of completion [5]. Hence the operating constraints are implemented in the following form

$$
\mathbf{C}_{1}(\mathrm{j}) \mathbf{x}_{\mathrm{k} \mid \mathrm{j}}+\mathbf{C}_{2}(\mathrm{j}) \mathbf{u}_{\mathrm{k} \mid \mathrm{j}}+\mathbf{C}_{3}(\mathrm{j}) \leq \mathbf{1 M}\left(1-\mathrm{y}_{\mathrm{k} \mid \mathrm{j}}\right)
$$

The following boundary conditions are also applied

$$
\begin{aligned}
\mathbf{x}_{\mathrm{k} \mid \mathrm{k}} & =\mathbf{A}_{p} \mathbf{x} \\
y_{\mathrm{k} \mid \mathrm{k}} & =1 \\
y_{\mathrm{k} \mid \mathrm{k}+\mathrm{N}+1} & =0
\end{aligned}
$$

where $\mathbf{x}$ denotes the current state estimate. The matrix $\mathbf{A}_{p}$ propagates the state forward by a short time period to account for the computation delays between the measurement of $\mathbf{x}$ and the execution of the first plan step [11]. The requirement for $y$ to start at 1 and end at 0 requires that the states pass through the target region at some step of the plan, thereby completing the mission.
MPC Application

This section describes the application of the MPC formulation above to the truck control problem in these experiments. For the rendezvous problem, the MPC trajectory optimization designs a path for the chaser truck relative to the target truck. The initial condition $\mathbf{x}$ in (15) is found by subtracting the GPS state estimate for the target from that of the chaser. The propagation $\mathbf{A}_{p}$ was for one second, roughly the amount of computation and communication delay in the system. The dynamics model in the relative frame is unchanged, apart from a translation of the velocity to enforce the maximum speed limit

$$
\forall j \in[k \ldots(k+N)] \mathbf{W}\left(\mathbf{v}_{k \mid j}+\mathbf{v}_{T}\right) \leq \mathbf{1} v_{\max }
$$

where $\mathbf{v}_{T}$ is the modeled target velocity. The system was discretized with a time-step of four seconds, using a zero-order hold. An offset was included to convert the relative velocity in the optimization to an absolute velocity before applying the approximate norm bound. The speed was limited to $0.8 \mathrm{~m} / \mathrm{s}$, the running speed of the truck for this experiment, and the force to less than $0.07 \mathrm{~N}$, equivalent to a maximum turning rate of approximately $5 \mathrm{deg} / \mathrm{s}$. This is well within the trucks operating limits.

The force reductions for robustness in (12) were $\beta_{1}=$ $0.03 \mathrm{~N}$ and $\beta_{2}=0.015 \mathrm{~N}$. The corresponding state perturbation was $\alpha=0.12 \mathrm{~m} / \mathrm{s}$. These values were derived from simulations using a nonlinear model of the truck dynamics, since it was anticipated that the model approximation would be the greatest source of uncertainty. Note that this implies that the disturbances are quite strong: the planned actuation is reduced to $35 \%$ of the total in the far future, along with a permitted $15 \%$ overspeed at the first plan step. The target region was a box extending from $0.5 \mathrm{~m}$ to $3.5 \mathrm{~m}$ east of the target truck (approximately behind it, given its nominal direction of travel to the west) with relative velocity less than $2 \mathrm{~cm} / \mathrm{s}$ in each component.

Given a solution to the trajectory optimization, the first force input $\mathbf{u}_{\mathrm{k} \mid \mathrm{k}}$ is converted to a steering angle. Its component perpendicular to the direction of travel is found using

$$
u_{\text {cross }}=\mathbf{e}_{\text {cross }} \cdot \mathbf{u}_{k \mid k}
$$

where the direction vector across the current track is found from the current velocity state $\mathbf{v}$ by

$$
\mathbf{e}_{\text {cross }}=\frac{1}{\|\mathbf{v}\|}\left(\begin{array}{cc}
0 & -1 \\
1 & 0
\end{array}\right) \mathbf{v}
$$


Then a scaling and bias conversion is performed to generate an integer servo command to be uploaded to the truck

$$
S_{\text {command }}=\operatorname{int}\left(a u_{\text {cross }}+b\right)
$$

The values of $a$ and $b$ are specific to each truck and were found experimentally. Their values were not known accurately, adding additional uncertainty to the problem. Note that the steering conversion (19) masks any longitudinal force command. The planner may call for small changes in speed, but these will be ignored. In summary, the rendezvous MPC scheme is as follows. At each time step:

1. Generate the relative state $\mathbf{x}$ from the current GPS estimates of the absolute states of each truck.

2. Solve the minimization of (11) subject to constraints (1), (9), (10) and (14)-(17).

3. Convert the first element of the continuous control to an equivalent steering servo command using (19)-(21) and upload this command to the truck.

4. Go to 1 .

\section{$\underline{\text { Rendezvous Tests }}$}

This section describes the hardware experiments using the MPC rendezvous controller. The test setup and procedure is described first. Then, the results are presented and discussed.

\section{$\underline{\text { Test Procedure }}$}

This section gives a step-by-step account of the procedure for each test.

1. Truck \# 1 (the chaser) was manually driven to the origin, marked by a cone. Its GPS estimator was started with its current position at $(0,0)$.

2. Truck \# 1 was manually driven to its starting position, roughly five meters away from the origin in the $\mathrm{Y}$ direction and pointing in the -X direction.

3. Truck \# 2 (the target) was manually driven to the origin. Its GPS estimator was started with its current position at $(0,0)$. Its alignment was approximately aiming in the $-\mathrm{X}$ direction. Note: the initial alignments of both trucks and the positioning of Truck \# 1 were performed manually, to a wide tolerance. The

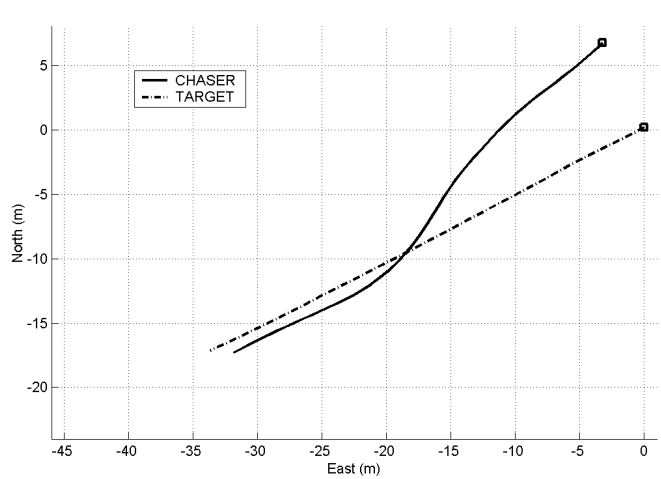

(a)

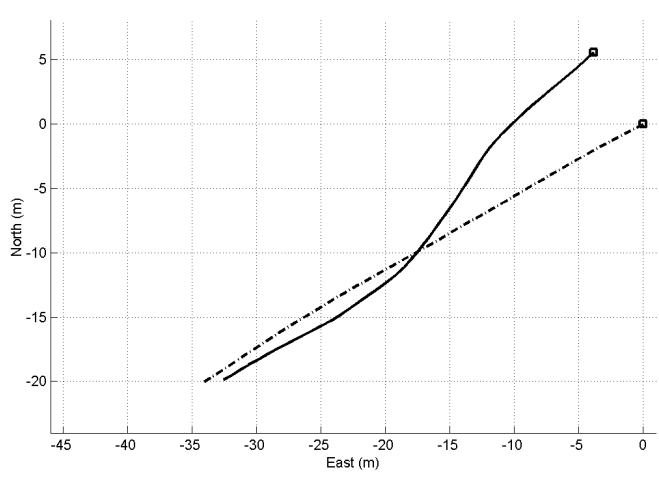

(b)

Fig. 5: Trajectories of Trucks for Two Tests

uncertainty of these quantities must be handled by the feedback control.

4. (Designated time $t=0$ ) Both trucks were given an initial command to drive straight ahead (i.e zero steering angle) with speed $0.8 \mathrm{~m} / \mathrm{s}$. (It is necessary for the trucks to be moving at the designated speed when the MPC controller is started. Otherwise, the point-mass dynamics model is invalid.)

5. (At time $t=5 \mathrm{~s}$ and at intervals of $4 \mathrm{~s}$ thereafter) The MPC controller solved the control problem from the current position of the trucks. Truck \# 1 was given a steering command derived from the resulting trajectory plan. Truck \# 2 was commanded to steer straight. Both trucks were commanded to remain at speed $0.8 \mathrm{~m} / \mathrm{s}$.

6. When the chaser truck was observed to have intercepted the target and matched its velocity, the test was terminated by the operator. 


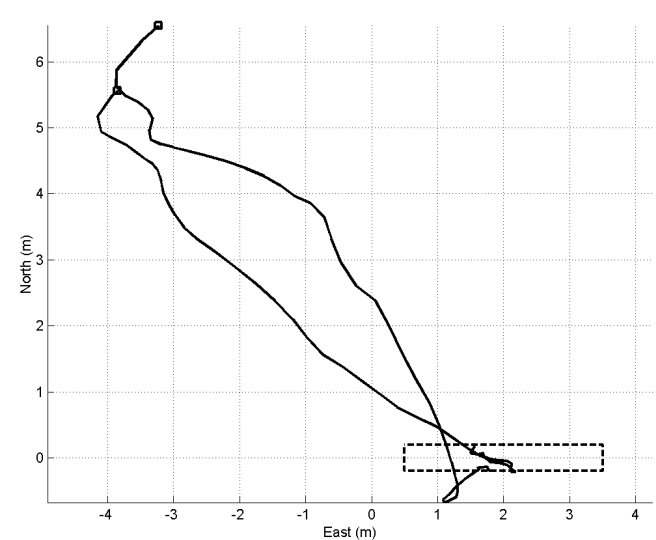

(a)
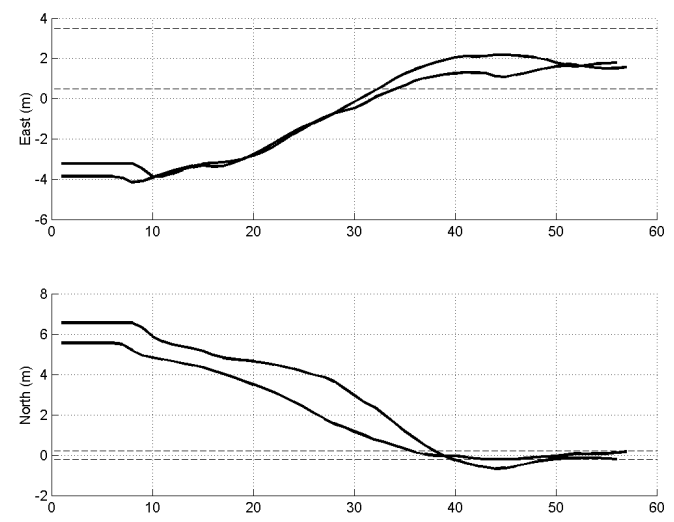

(b)

Fig. 6: Relative Motion of Trucks over Both Tests

$\underline{\text { Results }}$

Fig. 5 shows the trajectories of the two trucks on two different tests. Since this target truck is not under closed-loop control, its paths deviate due to initial misalignment and disturbances. In both cases, the chaser truck diverts from its initial path to come west of the target, then following it until the test is terminated. Some offset is observed between the target and chaser paths. Figs. 6(a) and 6(b) show the relative trajectories of the chaser with respect to the truck, both as X-Y plots and time histories. The dashed lines on each plot indicate the target region. In both tests, the chaser successfully reaches the target region.

The relative motion plots illustrate the strength of the disturbances at work. Typical plans would transfer smoothly from the starting position to the target region, but the plots show significant deviations. From observations of the tests, physical disturbances such as the effect of potholes have a significant effect on the results. Another substantial source of uncertainty is model error: the plan may call for small changes in speed, but only steering commands are used.

The importance of the robust feasibility modifications was apparent from prior simulations of these tests: without the robustness formulation, the MPC problem quickly became infeasible due to disturbances. For example, the optimization would fail if the truck exceeded its modeled maximum speed, as happens occasionally during the maneuver. In the tests, the optimization remained feasible throughout, demonstrating the success of the robustness formulation.

There is a slight overshoot observable in Fig. 6(b), but overall it would seem that any time delays present in the system have not had significant detrimental effects on the results. The forward propagation of the initial condition in (15) appears to have worked.

These results show that MILP/MPC can be used for real-time feedback control of a vehicle. The robust feasibility formulation enabled successful completion of rendezvous, even in the presence if significant uncertainty. Computation times were sufficiently short to maintain smooth control of the vehicle. Also, the linear model of a constant speed, limited turn vehicle e.g. a truck or aircraft is a workable approximation, allowing the benefits of linear optimization to be applied to practical control problems.

\section{CONCLUSIONS}

Experiments have been presented to demonstrate the use of Mixed-Integer Linear Programming for on-line replanning to control vehicles in the presence of dynamic uncertainty. Two schemes have been implemented on a ground vehicle testbed to demonstrate technologies aimed at UAV control. In one demonstration, a receding horizon formulation was used to maneuver a vehicle to its assigned goal with restricted detection of obstacles, representing limited-range radar. This architecture uses MILP for high-level path-planning, accounting for obstacle detection, while a low-level loop rejects disturbances to the vehicle. In the other demonstration, MILP was used as part of Model Predictive Control for the low-level control, to perform a rendezvous between two vehicles. This supported analytical claims of robustness and stability of the MPC formulation. This initial set of experiments will be extended to involve more complicated scenarios. This work will include the distribution of the MILP planning process across multiple computers and transition to a greater num- 


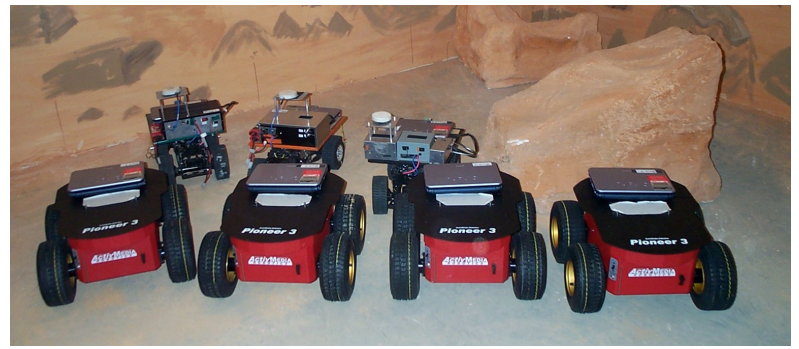

Fig. 7: New truck testbed developed for future experiments.

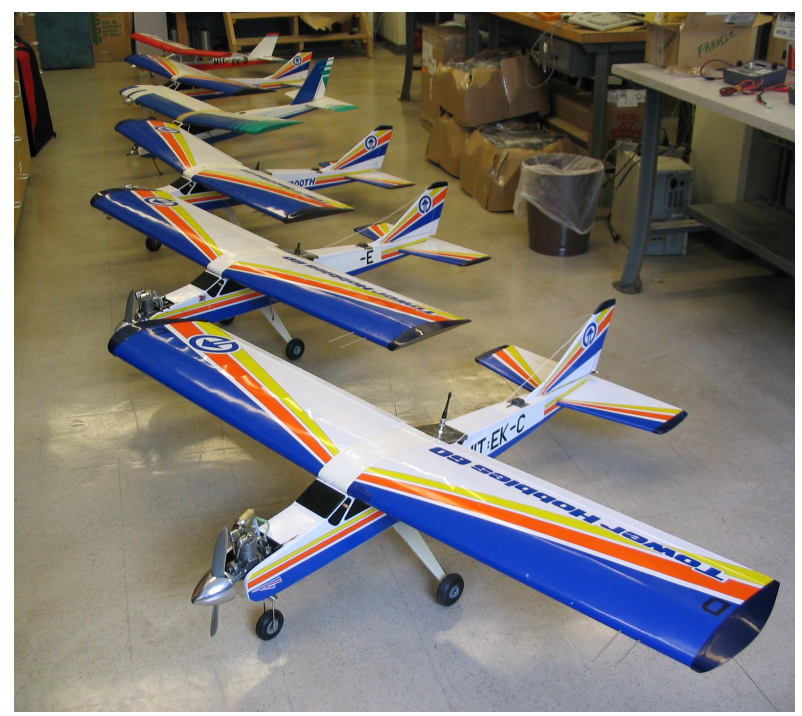

Fig. 8: Autonomous UAV testbed developed for future experiments.

ber and variation of vehicles. Figs. 7 and 8 show the new multi-truck and multi-UAV testbeds that have been developed to support this future work.

\section{ACKNOWLEDGMENTS}

Research funded under DARPA (MICA) contract N6601-01-C-8075. The testbeds were developed under the DURIP grant F49620-02-1-0216.

\section{REFERENCES}

[1] P. R. Chandler and M. Pachter, "Research Issues in Autonomous Control of Tactical UAVs," Proceedings of the American Control Conference, Philidelphia, PA, June 1998, IEEE, Washington, DC, pp. 394-398.

[2] J. S. Bellingham, "Receding Horizon Control of Autonomous Aerial Vehicles," ACC, May 2002.

[3] Y. Kuwata, Real-time Trajectory Design for Unmanned Aerial Vehicles using Receding Horizon
Control, S.M. Thesis, Dept. of Aeronautics and Astronautics, MIT, June 2003.

[4] A. G. Richards, "Trajectory Optimization using Mixed-Integer Linear Programming," Masters Thesis, Massachusetts Institute of Technology, June 2002 .

[5] A. G. Richards, J. P. How, "Model Predictive Control of Vehicle Maneuvers with Guaranteed Completion Time and Robust Feasibility," submitted to ACC 2003.

[6] J.M. Maciejowski, Predictive Control with Constraints, Prentice Hall, England, 2002.

[7] A. Richards, T. Schouwenaars, J. How, E. Feron, "Spacecraft Trajectory Planning With Collision and Plume Avoidance Using Mixed-Integer Linear Programming," Journal of Guidance, Control and Dynamics, AIAA, August 2002.

[8] A. G. Richards, J. P. How, "Aircraft Trajectory Planning with Collision Avoidance using Mixed Integer Linear Programming," ACC, May 2002.

[9] T. Schouwenaars, B. DeMoor, E. Feron and J. How, "Mixed integer programming for safe multi-vehicle cooperative path planning," ECC, Porto, Portugal, September 2001.

[10] A. Bemporad and M. Morari, "Control of Systems Integrating Logic, Dynamics, and Constraints," in Automatica, Pergamon / Elsevier Science, Vol. 35, pp. 407-427, 1999.

[11] R. Franz, M. Milam, and J. Hauser, "Applied Receding Horizon Control of the Caltech Ducted Fan," ACC 2002.

[12] W. B. Dunbar, M. B. Milam, R. Franz and R. M. Murray, "Model Predictive Control of a Thrust-Vectored Flight Control Experiment," accepted for 15th IFAC World Congress on Automatic Control, 2002

[13] W. Dunbar and R. Murray, "Model predictive control of coordinated multi-vehicle formations," IEEE $C D C, 2002$.

[14] L. Cremean, W. Dunbar, D. van Gogh, J. Hickey, E. Klavins, J. Meltzer and R. Murray, "The Caltech Multi-Vehicle Wireless Testbed," IEEE CDC, 2002.

[15] R. Fourer, D. M. Gay, and B. W. Kernighar, AMPL, A modeling language for mathematical programming, The Scientific Press, 1993.

[16] ILOG CPLEX User's guide, ILOG, 1999.

[17] N. A. Pohlman, "Estimation and Control of a Multi-Vehicle Testbed Using GPS Doppler Sensing," S.M. Thesis, MIT, 2002.

[18] H. P. Rothwangl, "Numerical Synthesis of the Time Optimal Nonlinear State Controller via Mixed Integer Programming," IEEE ACC, 2001. 